\title{
Crystal growth and property characterization for PIN-PMN-PT ternary piezoelectric crystals
}

\author{
Jian Tian* and Pengdi Han \\ H.C. Materials Corporation \\ 479 Quadrangle Dr., Suite-E, Bolingbrook, IL 60440, USA \\ *jtian@hcmat.com
}

Received 18 July 2013; Accepted 20 October 2013; Published 9 December 2013

\begin{abstract}
Binary piezoelectric crystal $\mathrm{Pb}\left(\mathrm{Mg}_{1 / 3} \mathrm{Nb}_{2 / 3}\right) \mathrm{O}_{3}-\mathrm{PbTiO}_{3}(\mathrm{PMN}-\mathrm{PT})$ has excellent dielectric and piezoelectric properties, which has led to the commercialization for medical ultrasound imaging. Recently, there is a growing demand for piezoelectric crystals with improved thermal and electrical properties. Ternary piezoelectric crystals $\mathrm{Pb}\left(\mathrm{In}_{1 / 2} \mathrm{Nb}_{1 / 2}\right) \mathrm{O}_{3}-\mathrm{Pb}\left(\mathrm{Mg}_{1 / 3} \mathrm{Nb}_{2 / 3}\right) \mathrm{O}_{3}-\mathrm{PbTiO}_{3}(\mathrm{PIN}-$ PMN-PT) have increased depoling temperature and coercive field than binary PMN-PT. To better understand the ternary crystal system and improve crystal properties, we systematically investigated crystal growth of PIN-PMN-PT with modified Bridgman method. Like PMN-PT, PIN-PMN-PT crystals have excellent piezoelectric properties (e.g., $k_{33} \sim 0.87-0.92, d_{33} \sim 1000$ $2200 \mathrm{pm} / \mathrm{V})$. Higher PIN content leads to $\sim 40^{\circ} \mathrm{C}$ increase in depoling temperature and more than doubling of coercive field $(\sim 7.0 \mathrm{kV} / \mathrm{cm})$. Such improvements are advantageous for applications where high temperature and/or high-drive are needed.
\end{abstract}

Keywords: PIN-PMN-PT; ternary crystals; coercive field; thermal stability.

\section{Introduction}

The excellent dielectric and piezoelectric properties of $\mathrm{Pb}\left(\mathrm{Mg}_{1 / 3} \mathrm{Nb}_{2 / 3}\right) \mathrm{O}_{3}-\mathrm{PbTiO}_{3}$ (PMN-PT) single crystal have led to a successful commercialization in advanced medical ultrasound. ${ }^{1}$ Over the past decade, significant progresses have been achieved on crystal growth and characterization, ${ }^{2-6}$ and new applications ${ }^{7-15}$ for binary PMN-PT crystals.

Despite the excellent properties of PMN-PT single crystals, the relatively low depoling temperature $\left(T_{\mathrm{R} / \mathrm{T}} \sim 75-\right.$ $\left.97^{\circ} \mathrm{C}\right)$, low coercive field $\left(E_{C} \sim 2.5 \mathrm{kV} / \mathrm{cm}\right)$ and low mechanical quality factor $\left(Q_{M} \sim 80\right)$ remain concerns for many applications especially those require high drive conditions. A low $T_{\mathrm{R} / \mathrm{T}}$ limits application temperature and generally leads to a large temperature-dependent variations in crystal property and thus transducer performance. In addition, $E_{C}$ decreases as temperature increases when transducer operates. Low $Q_{M}$ leads to more heat generation and higher temperature rise during transducer operation. Thus, higher coercive field and improved mechanical quality factor of piezocrystals are especially important for high drive applications. Recently, piezocrystals with improved thermal and electrical properties have become active in research. ${ }^{16-21} \mathrm{PMN}-\mathrm{PT}$ based ternary $\mathrm{Pb}\left(\mathrm{In}_{1 / 2} \mathrm{Nb}_{1 / 2}\right) \mathrm{O}_{3}-\mathrm{Pb}\left(\mathrm{Mg}_{1 / 3} \mathrm{Nb}_{2 / 3}\right) \mathrm{O}_{3}-\mathrm{PbTiO}_{3}$ (PIN-PMNPT) crystals are attractive because (1) PIN-PMN-PT crystals have improved thermal and electrical properties; and (2) high quality and large-size PIN-PMN-PT crystals can be grown with various compositions/properties. ${ }^{21-28}$

In this paper, we report crystal growth and property characterization for ternary PIN-PMN-PT crystals. Crystals of various starting compositions were grown. Dielectric, piezoelectric, and elastic properties were measured on specimens selected along crystal growth direction. The goals are to investigate crystal growth behavior, characterize property variation, and improve understanding of the compositionproperty relationship for the ternary PIN-PMN-PT crystals.

\section{Experimental}

Like PMN-PT, ternary PIN-PMN-PT crystals were grown with a modified Bridgman method. Starting compositions for crystal growth were chosen in the rhombohedral phase region close to the morphotropic phase boundary (MPB, Fig. 1) for enhanced piezoelectric properties. ${ }^{21-28}$ Various PIN contents from $12 \%$ to $60 \%$ for PIN-PMN-PT ternary compositions, as well as the binary composition of PIN-0.31PT, were prepared for crystal growth experiments. Detailed chemicals, precursor preparation and crystal growth information were reported earlier. $^{23}$

$\{001\}$ crystal plates and bars were prepared to characterize crystal properties in [001] direction, ${ }^{6,23}$ Residual stresses from crystal growth and mechanical finishing were relieved by annealing at $\sim 600^{\circ} \mathrm{C}$. Chromium/gold electrodes were sputter coated on appropriate faces of specimens. Samples were poled at up to $10 \mathrm{kV} / \mathrm{cm}$ in air at room temperature.

Properties were measured at room temperature of $24 \mathrm{~h}$ after poling. Measurement procedures for crystal properties, including the free and clamped dielectric constants $\left(K_{33}^{T}\right.$ and $\left.K_{33}^{S}\right)$, electromechanical coupling factors $\left(k_{33}\right.$ and $\left.k_{t}\right)$, elastic

This is an Open Access article published by World Scientific Publishing Company. It is distributed under the terms of the Creative Commons Attribution 3.0 (CC-BY) License. Further distribution of this work is permitted, provided the original work is properly cited. 


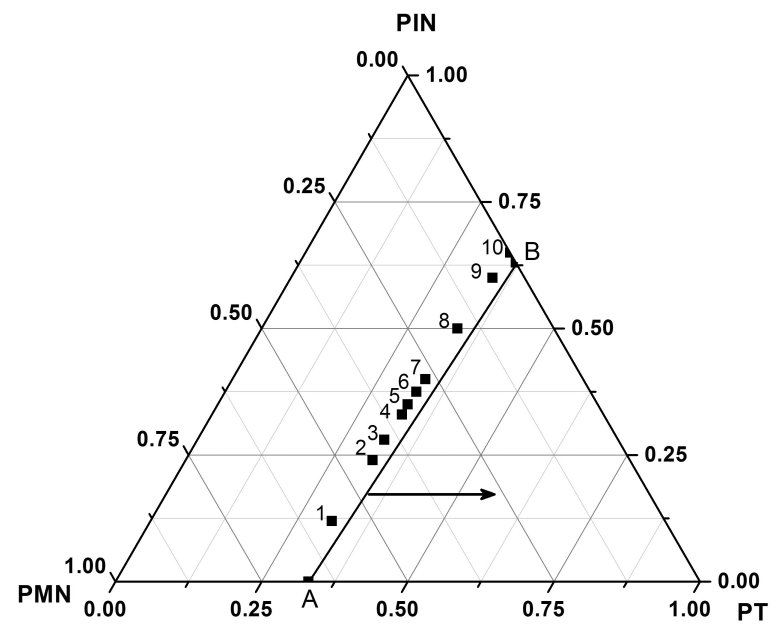

Fig. 1. Starting compositions (1-10) for crystal growth. Arrow indicates the trend of compositional change along crystal grow direction. Line $\mathrm{AB}$ is a linear approximation to the MPB composition for ternary PIN-PMN-PT.

compliance $\left(s_{33}^{E}\right)$, ferroelectric hysteresis, field-induced strain, $E_{C}$ and large-signal piezoelectric strain coefficient $\left(d_{33}\right)$, were reported earlier. ${ }^{6,23}$ Measurement of $Q_{M}$ followed IEEE standard. ${ }^{29}$ The $T_{\mathrm{R} / \mathrm{T}}$ and Curie temperature $T_{C}$ were measured on a custom thermal testing system with $0.1{ }^{\circ} \mathrm{C}$ accuracy. The chemical composition of crystal samples were analyzed with an inductively coupled plasma (ICP) method.

\section{Results and Discussions}

Crystal growth experiments demonstrated that PIN-PMN-PT crystals of various starting compositions can grow directly from stoichiometric melts with modified Bridgman method. High quality and large size (up to 4 " diameter) PIN-PMNPT single crystals of perovskite phase have been successfully grown for PIN content of 12 to $33 \mathrm{~mol} \%$. Thus, ternary crystals offer a broad selection of properties for transducer applications that cannot be realized for PMN-PT. For compositions with higher PIN content ( $>33 \mathrm{~mol} \%)$, growth flux was needed to obtain perovskite phase single crystals. However, it is more challenging to obtain high quality crystal with flux method because flux can be trapped in the as-grown crystal to form inclusions. In addition, flux dilutes the ions for crystal growth, thus flux method generally has a much slower growth rate than the stoichiometric melt growth. Like PMNPT, PIN-PMN-PT crystals can grow directly from stoichiometric melt. This is critical for the commercialization of PIN-PMN-PT single crystals.

To determine the growth behavior of ternary PIN-PMNPT single crystals, samples were selected along crystal growth direction for ICP analyses. Chemical composition of crystal samples were then calculated from the ICP data. Figure 2 showed variation in the end-member components for 0.24PIN-PMN-PT and 0.33PIN-PMN-PT crystals. Both

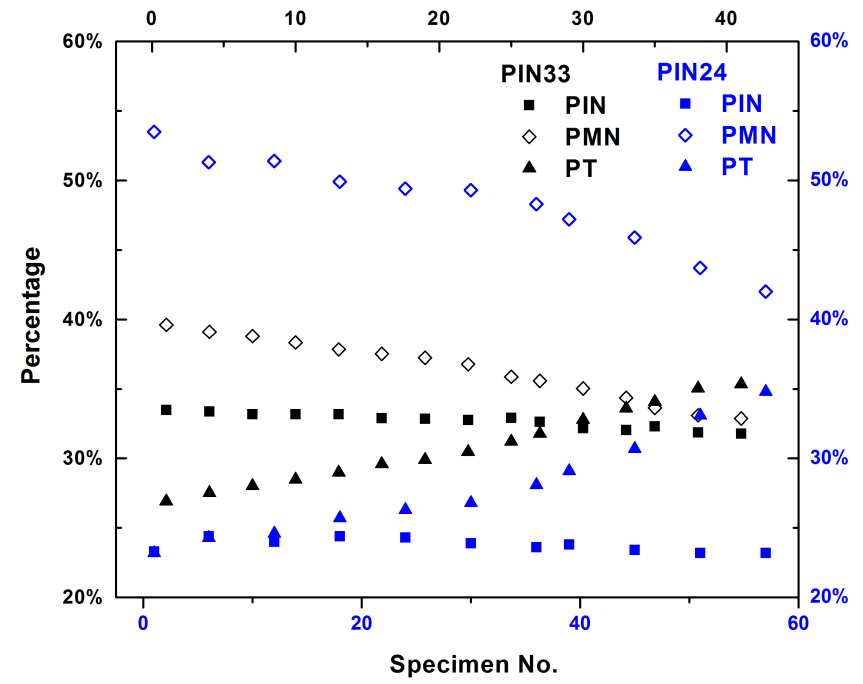

Fig. 2. (Color online) Changes in composition along growth direction for ternary crystals of PIN24-PMN-PT (blue color) and PIN33-PMN-PT (black color). Specimens are $1 \mathrm{~mm}$ apart with No. 1 from crystal base.

crystals show similar trend, with increasing PT and decreasing PMN along crystal growth direction. The increase in PT can be largely accounted by the decrease in PMN. In contrast, PIN content only had a slightly decreasing trend for both crystals, suggesting that PIN is least sensitive to chemical segregation during crystal growth. In addition, changes in PIN content do not seem to affect much of the segregation behavior in PMN and PT. This is important for the composition/property optimization of ternary PIN-PMN-PT single crystals.

As PIN content increases, the $T_{\mathrm{R} / \mathrm{T}}$ of PIN-PMN-PT crystals generally increase. Figure 3 illustrates the relationship between the maximum $T_{\mathrm{R} / \mathrm{T}}$ value and PIN content of different crystals. The $T_{\mathrm{R} / \mathrm{T}}-\mathrm{PIN} \%$ relationship fits well to exponential growth $\left(R^{2}>0.99\right) . T_{\mathrm{R} / \mathrm{T}}$ increased noticeably for PIN-PMN-PT crystals of high PIN content. The

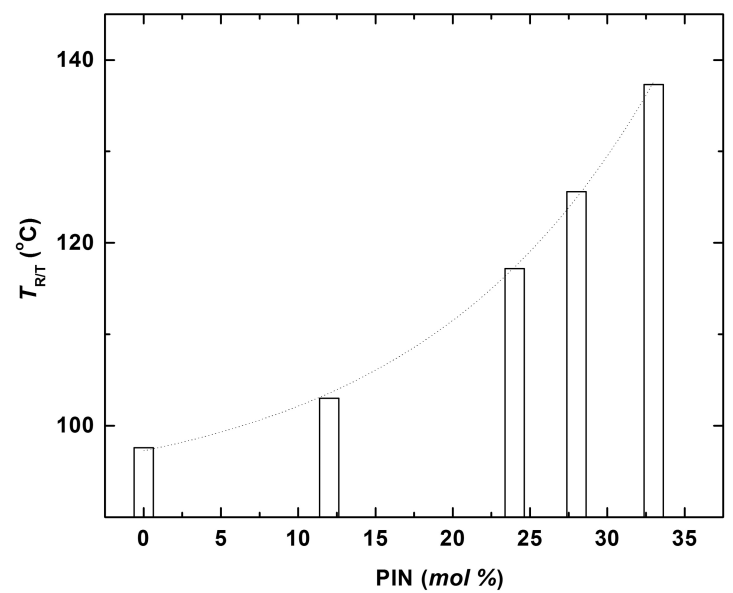

Fig. 3. Relationship between maximum $T_{\mathrm{R} / \mathrm{T}}$ in a crystal boule and PIN content in starting composition. Dotted line is an exponential fit. 


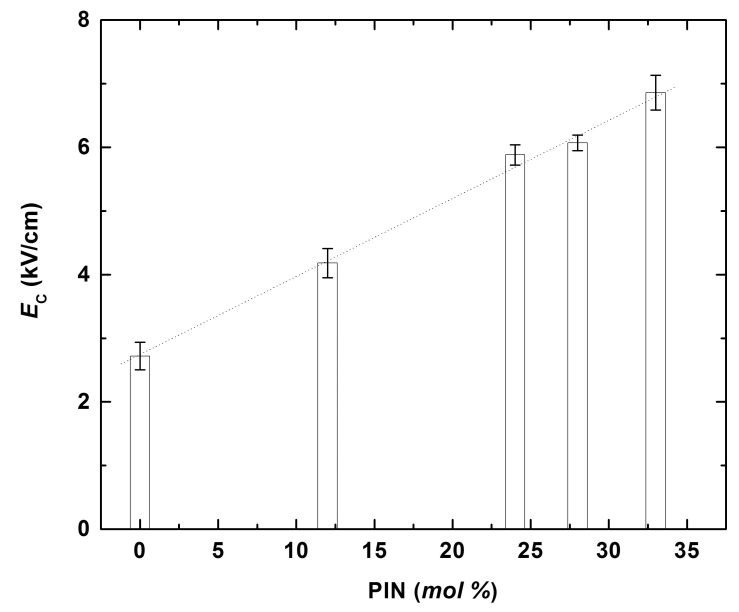

Fig. 4. Relationship of average $E_{C}$ in a crystal boule and PIN content in starting composition. Error bars show standard deviation of $E_{C}$. Dotted line is a linear fit to $E_{C}-\mathrm{PIN} \%$.

maximum $T_{\mathrm{R} / \mathrm{T}}$ of $0.33 \mathrm{PIN}-\mathrm{PMN}-\mathrm{PT}$ is $137^{\circ} \mathrm{C}$, which is $20^{\circ} \mathrm{C}$ over $0.24 \mathrm{PIN}-\mathrm{PMN}-\mathrm{PT}$ and $40^{\circ} \mathrm{C}$ over PMN-PT. For both PMN-PT and PIN-PMN-PT single crystals, $T_{\mathrm{R} / \mathrm{T}}$ is the upper temperature limit for piezoelectric application. Crystal will depole at temperatures above $T_{\mathrm{R} / \mathrm{T}}$ and properties degrade rapidly. Higher $T_{\mathrm{R} / \mathrm{T}}$ improves allows crystal application at a higher temperature without risking of thermal depoling. Thus, reliability of crystal transducers is enhanced with increased $T_{\mathrm{R} / \mathrm{T}}$. Furthermore, increased $T_{\mathrm{R} / \mathrm{T}}$ also improves transducer performance since temperature induced variation in crystal property is significantly reduced.

Similar to the $T_{\mathrm{R} / \mathrm{T}}, E_{C}$ values of PIN-PMN-PT crystals increase with PIN content. Average value of $E_{C}$ was plot (Fig. 4) since $E_{C}$ is relatively stable within a crystal. $E_{C}$ exhibits a good linear relationship with PIN mol\% $\left(R^{2}>0.99\right)$. Higher $E_{C}$ allows a greater driving electric field in high power transducer application. In addition, enhanced $E_{C}$ eliminates/reduces the need for a bias voltage that is commonly required for binary PMN-PT crystal in high drive application, which improves the robustness of crystal transducers. $E_{C}$ is relatively stable within a crystal boule for PIN-PMN-PT crystals of different PIN contents (e.g., 12, 24, 28 and $33 \mathrm{~mol} \%$ ). The moderately steady PIN content along crystal growth direction (Fig. 2) likely led to the stable coercivity for both $0.24 \mathrm{PIN}-\mathrm{PMN}-\mathrm{PT}$ and 0.33PIN-PMNPT crystals. This is likely true for PIN-PMN-PT crystals of other PIN contents. In contrast, variations in PMN and PT contents had little effect on coercivity of ternary PIN-PMNPT crystals, consistent with the binary PMN-PT single crystal.

Other key piezoelectric properties of ternary PIN-PMNPT crystals, such as electromechanical coupling factors $k_{33}$ and $k_{t}$, and piezoelectric strain coefficient $d_{33}$, remain excellent. On the other hand, PIN-PMN-PT crystals generally have lower dielectric values than PMN-PT, with decreasing dielectric values with increasing PIN content. Improvements

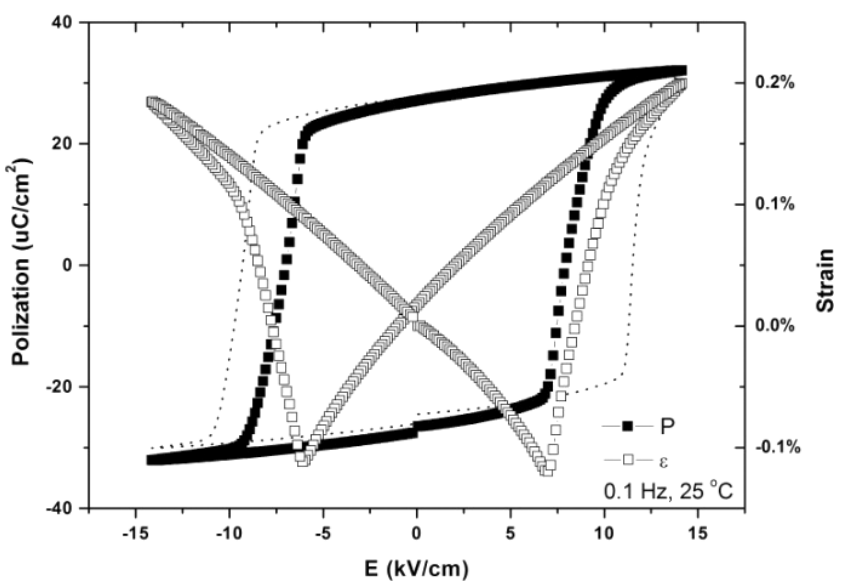

Fig. 5. Ferroelectric hysteresis an piezoelectric strain response for Mn modified PIN-PMN-PT single.

in $T_{\mathrm{R} / \mathrm{T}}$ and $E_{C}$ of PIN-PMN-PT crystals further expands operating conditions and enhanced reliability for crystal transducers.

The coercive field of Mn modified PIN-PMN-PT crystal increased (Fig. 5). The $E_{C}$ value based on the first hysteresis measurement is $\sim 11 \mathrm{kV} / \mathrm{cm}$. The $E_{C}$ value after 10 hysteresis measurements is $\sim 8 \mathrm{kV} / \mathrm{cm}$. Both values are higher than those of unmodified PIN-PMN-PT crystal. In addition, Mn modified ternary PIN-PMN-PT crystals show improvement in $Q_{M}$, with values up to 700 . However, other important properties including coupling factors, piezoelectric coefficient and dielectric constant were noticeably degraded.

ICP analyses revealed significant compositional segregation of Mn for Mn modified PIN-PMN-PT crystals. As shown in Fig. 6, the segregation of Mn is more than twice of the PT, which presents a challenge for crystal growth. New crystal growth technique with continuous material feeding

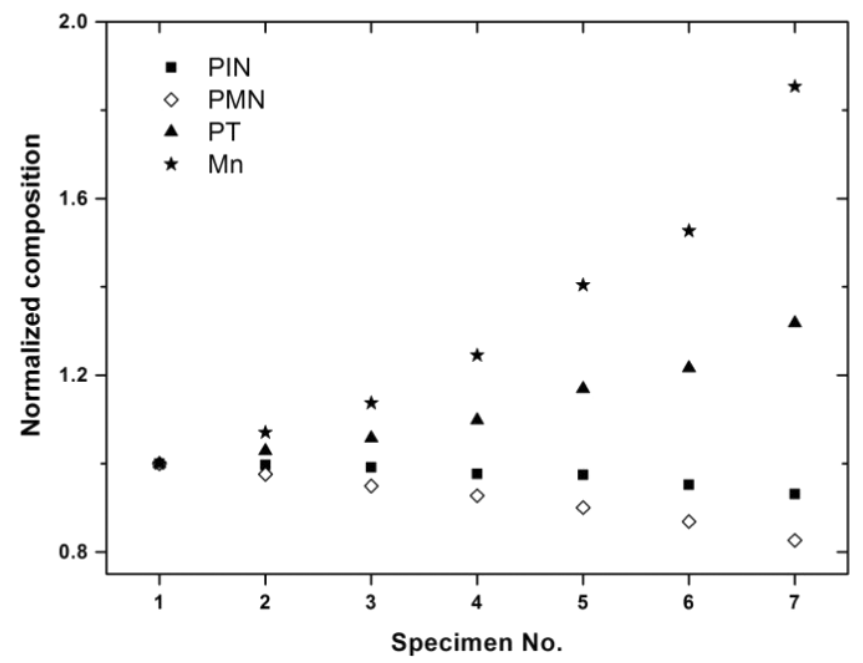

Fig. 6. Composition segregation along crystal growth direction for Mn modified PIN-PMN-PT single. Samples 1-7 were selected from a crystal boule along growth direction, with sample 1 from base and 7 near top. 
during crystal growth is needed to improve the control of compositional segregation for $\mathrm{Mn}$ modified PIN-PMN-PT crystals.

\section{Conclusion}

Ternary PIN-PMN-PT crystals with PIN content up to $33 \mathrm{~mol} \%$ can be grown directly from stoichiometric melt with modified Bridgman method. PIN-PMN-PT crystals have improved depoling temperature and coercive field, with $T_{\mathrm{R} / \mathrm{T}}$ up to $137^{\circ} \mathrm{C}$ and $E_{C}$ up to $7.0 \mathrm{kV} / \mathrm{cm}$ for PIN33-PMN-PT. Other key piezoelectric properties including coupling factors $k_{33}, k_{t}$ and piezoelectric coefficient $d_{33}$ remain excellent. These improvements are important especially for high-drive transducers.

Mn-modified PIN-PMN-PT crystals show improved coercive field and mechanical quality factor at the expense of degraded electromechanical coupling, piezoelectric and dielectric properties. In addition, Mn has significant segregation during crystal growth.

\section{Acknowledgments}

This research was supported by ONR contracts N00014-C and N00014-M, and HC-R\&D fund-2001-13.

\section{References}

${ }^{1}$ J. Chen and R. Panda, Review: Commercialization of piezoelectric single crystals for medical imaging applications, 2005 IEEE IUS Proc., ed. M. P. Yuhas (IEEE, Rotterdam, Netherlands, 2005), pp. 235-240.

${ }^{2}$ P. Han, J. Tian and W. Yan, Handbook of Advanced Dielectric, Piezoelectric and Ferroelectric Materials: Synthesis, Properties and Applications, ed. Z. G. Ye (Woodhead Publishing, 2008), p. 1096.

${ }^{3}$ S. E. Park and T. R. Shrout, Characteristics of relaxor-based piezoelectric single crystals for ultrasonic transducers, IEEE Trans. Ultrason. Ferroelectr. Freq. Control 44, 1140 (1997).

${ }^{4}$ H. S. Luo, G. S. Xu, H. Q. Xu, P. C. Wang and Z. W. Yin, Compositional homogeneity and electrical properties of lead magnesium niobate titanate single crystals grown by a modified Bridgman technique, Jpn. J. Appl. Phys., Part 1 39, 5581 (2000).

${ }^{5}$ A. A. Bokov and Z. G. Ye, Ferroelectric properties of monoclinic $\mathrm{Pb}\left(\mathrm{Mg}_{1 / 3} \mathrm{Nb}_{2 / 3}\right) \mathrm{O}_{3}-\mathrm{PbTiO}_{3}$ crystals, Phys. Rev. B 66, 094112 (2002).

${ }^{6}$ J. Tian, P. D. Han and D. A. Payne, Measurements along the growth direction of PMN-PT crystals: Dielectric, piezoelectric, and elastic properties, IEEE Trans. Ultrason. Ferroelectr. Freq. Control 54, 1895 (2007).

${ }^{7}$ J. Chen, A. Shurland, J. Perry, B. Ossmann and T. R. Gururaja, Electrostrictive transducers for medical ultrasonic applications, Proc. 10th IEEE ISAF, eds. B. M. Kulwicki, A. Amin and A. Safari (IEEE, East Brunswich, NJ, 1996), pp. 27-30.

${ }^{8}$ J. R. Yuan, X. Jiang, P. J. Cao, A. B. Sadaka, R. K. Snook and P. W. Rehirg, High frequency piezo composites microfabricated ultrasound transducers for intravascular imaging, 2006 IEEE IUS Proc., ed. M. P. Yuhas (IEEE, Vancouver, BC, Canada, 2006), p. 5. ${ }^{9}$ Q. Zhou, X. Xu, E. J. Gottlieb, L. Sun, J. M. Cannata, H. Ameri, M. S. Humayun, P. Han and K. K. Shunk, PMN-PT single crystal, high-frequency ultrasonic needle transducers for pulsed-wave doppler application, IEEE Trans. Ultrason. Ferroelectr. Freq. Control 54, 668 (2007).

${ }^{10} \mathrm{~S}$. M. Rhim and H. Jung, Piezoelectric single crystal for medical ultrasound transducer, 2007 IEEE IUS Proc., ed. M. P. Yuhas (IEEE, New York City, NY, 2007), p. 5.

${ }^{11}$ X. Jiang, K. Snook, T. Walker, A. Portune, R. Harber, X. Geng, J. Welter and W. Hackenberger, Single crystal piezoelectric composite transducers for ultrasound NDE applications, 2008 Proc. SPIE (San Diego, CA), pp. 69340D-1-10.

${ }^{12}$ J. M. Powers, M. B. Moffett and F. Nussbaum, Single crystal naval transducer development, Proc. 12th IEEE ISAF, eds. S. K. Streiffer, B. J. Gibbons and T. Tsurumi (IEEE, Honolulu, HI, 2000), pp. 351-354.

${ }^{13}$ K. Snook, P. W. Rehirg, X. Jiang, W. S. Hackenberger, R. J. Meyer and D. Markley, Advanced piezoelectric single crystal based transducers for naval sonar applications, 2005 IEEE IUS Proc., ed. M. P. Yuhas (IEEE, Rotterdam, Netherlands, 2005), pp. 1065-1068.

${ }^{14}$ M. B. Moffett, H. C. Robinson, J. M. Powers and P. D. Baird, Single-crystal lead magnesium niobate-lead titanate (PMN/PT) as a broadband high power transduction material, J. Acoust. Soc. Am. 121, 2591 (2007).

${ }^{15}$ A. Amin, E. McLaughlin, H. Robinson and L. Ewart, Mechanical and thermal transitions in morphotropic PZN-PT and PMN-PT single crystals and their implication for sound projectors, IEEE Trans. Ultrason. Ferroelectr. Freq. Control 54, 1090 (2007).

${ }^{16}$ S. Zhang, P. W. Rehirg, C. A. Randall and T. R. Shrout, Crystal growth and electrical properties of $\mathrm{Pb}\left(\mathrm{Yb}_{1 / 2} \mathrm{Nb}_{1 / 2}\right) \mathrm{O}_{3}-\mathrm{PbTiO}_{3}$ perovskite single crystals, J. Cryst. Growth 234, 415 (2002).

${ }^{17}$ S. Zhang, L. Lebrun, S. Rhee, R. E. Eitel, C. A. Randall and T. R. Shrout, Crystal growth and characterization of new high curie temperature $(1-x) \mathrm{BiScO}_{3}-x \mathrm{PbTiO}_{3}$ single crystals, J. Cryst. Growth 236, 210 (2002).

${ }^{18}$ Y. H. Bing and Z. G. Ye, Effects of growth conditions on the domain structure and dielectric properties of $(1-x) \mathrm{Pb}\left(\mathrm{Sc}_{1 / 2} \mathrm{Nb}_{1 / 2}\right)$ $\mathrm{O}_{3}-x \mathrm{PbTiO}_{3}$ single crystals, Mat. Sci. Eng. B 120, 72 (2005).

${ }^{19}$ N. Yasuda, H. Ohwa, M. Kume, K. Hayashi, Y. Hosono and Y. Yamashita, Crystal growth and electrical properties of lead indium niobate-lead titanate binary single crystal, J. Cryst. Growth 229, 299 (2001).

${ }^{20}$ T. Karaki, M. Nakamoto, Y. Sumiyoshi, M. Adachi, Y. Hosono and Y. Yamashita, Top-seeded solution growth of $\mathrm{Pb}\left[\left(\mathrm{In}_{1 / 2} \mathrm{Nb}_{1 / 2}\right)\right.$, $\left.\left(\mathrm{Mg}_{1 / 3} \mathrm{Nb}_{2 / 3}\right), \mathrm{Ti}\right] \mathrm{O}_{3}$ single crystals, Jpn. J. Appl. Phys., 1 42, 6059 (2003).

${ }^{21}$ Y. Hosono, Y. Yamashita, H. Sakamoto and N. Ichinose, Growth of single crystals of high-curie-temperature $\mathrm{Pb}\left(\mathrm{In}_{1 / 2} \mathrm{Nb}_{1 / 2}\right) \mathrm{O}_{3}-\mathrm{Pb}$ $\left(\mathrm{Mg}_{1 / 3} \mathrm{Nb}_{2 / 3}\right) \mathrm{O}_{3}-\mathrm{PbTiO}_{3}$ ternary systems near morphotropic phase boundary, Jpn. J. Appl. Phys., 1 42, 5681 (2003).

${ }^{22} \mathrm{G}$. Xu, K. Chen and D. F. Yang, Growth and electrical properties of large size $\mathrm{Pb}\left(\mathrm{In}_{1 / 2} \mathrm{Nb}_{1 / 2}\right) \mathrm{O}_{3}-\mathrm{Pb}\left(\mathrm{Mg}_{1 / 3} \mathrm{Nb}_{2 / 3}\right) \mathrm{O}_{3}-\mathrm{PbTiO}_{3}$ crystals prepared by the vertical Bridgman technique, Appl. Phys. Lett. 90, 032901 (2007).

${ }^{23}$ J. Tian, P. Han, X. Huang, H. Pan, J. F. Carroll and D. A. Payne, Improved stability for piezoelectric crystals grown in the lead indium niobate-lead magnesium niobate-lead titanate system, Appl. Phys. Lett. 91, 222903 (2007). 
${ }^{24}$ J. Tian, P. Han, J. F. Carroll and D. A. Payne, Improved properties of piezoelectric crystal lead indium niobate-lead magnesium niobatelead titanate, 2008 IEEE IUS Proc., ed. K. R. Waters (IEEE, Beijing, China, 2008), pp. 269-271.

${ }^{25}$ J. Luo, W. Hackenberger, S. Zhang and T. Shrout, Piezoelectric and dielectric properties of PIN-PMN-PT crystals grown by Bridgman method, 2008 IEEE IUS Proc., ed. K. R. Waters (IEEE, Beijing, China, 2008), pp. 261-264.

${ }^{26}$ J. Luo, W. Hackenberger, S. Zhang and T. Shrout, The progress update of relaxor piezoelectric single crystals, 2009 IEEE IUS Proc., ed. M. P. Yuhas (IEEE, Rome, Italy, 2009), pp. 968-971.

${ }^{27}$ J. Tian, P. Han, J. F. Carroll and D. A. Payne, Growth and improved properties of ternary piezoelectric single crystals in lead indium niobate-lead magnesium niobate-lead titanate system, 2009 IEEE IUS Proc., ed. M. P. Yuhas (IEEE, Rome, Italy, 2009), pp. 972-975.

${ }^{28}$ S. Zhang, F. Li, N. P. Sherlock, J. Luo, H. J. Lee, R. Xia, R. J. Meyer, Jr., W. Hackenberger and T. R. Shrout, Recent developments on high curie temperature PIN-PMN-PT ferroelectric crystals, J. Cryst. Growth 318, 846 (2011).

${ }^{29}$ Standards committee of the IEEE ultrasonics, ferroelectrics, and frequency control society, IEEE Standard on Piezoelectricity (The Institute of Electrical and Electronics Engineers Inc., New York, 1987), pp. 1-66. 